\title{
A nonlinear general Neumann problem involving two critical exponents
}

\author{
Rejeb Hadiji* and Habib Yazidi ${ }^{\dagger} *$
}

\begin{abstract}
We discuss the existence of solutions to the following nonlinear problem involving two critical Sobolev exponents

$$
\begin{cases}-\operatorname{div}(p(x) \nabla u)=\beta|u|^{2^{*}-2} u+f(x, u) & \text { in } \Omega \\ u \neq 0 & \text { in } \Omega \\ \frac{\partial u}{\partial \nu}=Q(x)|u|^{2_{*}-2} u & \text { on } \partial \Omega\end{cases}
$$

where $\beta \geq 0, Q$ is continuous on $\partial \Omega, p \in H^{1}(\Omega)$ is continuous and positive in $\bar{\Omega}$ and $f$ is a lower-order perturbation of $|u|^{2^{*}-1}$ with $f(x, 0)=0$.

Keywords : Sobolev critical exponent, The trace embedding, Variational problem, Critical nonlinearity in the boundary, Palais-Smale Condition, The mean curvature.
\end{abstract}

2010 AMS subject classifications: 35J20, 35J25, 35J60.

\section{Introduction}

In this work, we deal with the following problem

$$
\left\{\begin{array}{cl}
-\operatorname{div}(p(x) \nabla u)=\beta|u|^{2^{*}-2} u+f(x, u) & \text { in } \Omega, \\
u \neq \equiv 0 & \text { in } \Omega, \\
\frac{\partial u}{\partial \nu}=Q(x)|u|^{2_{*}-2} u & \text { on } \partial \Omega,
\end{array}\right.
$$

where $\Omega \subset \mathbb{R}^{N}, N \geq 3$, is a bounded domain with the smooth boundary $\partial \Omega, \nu$ is the outer normal on $\partial \Omega, \beta \geq 0$ is a constant, the coefficient $Q$ is continuous on $\partial \Omega$, the coefficient $p \in H^{1}(\Omega)$ is continuous and positive in $\bar{\Omega}$ and $f(x, u): \Omega \times \mathbb{R} \rightarrow \mathbb{R}$ is measurable in $x$, continuous in $u$.

Here, $2_{*}=\frac{2(N-1)}{N-2}$ is the critical Sobolev exponent for the trace embedding of the space $H^{1}(\Omega)$ into $L^{2 *}(\partial \Omega)$ and $2^{*}=\frac{2 N}{N-2}$ is the critical Sobolev exponent for the embedding $H^{1}(\Omega)$ into $L^{2^{*}}(\Omega)$. Both embedding are continuous, but not compact. Our goal is to

${ }^{*}$ Université Paris-Est, Laboratoire d'Analyse et de Mathématiques Appliquées (LAMA), CNRS : UMR 8050, 61, Avenue du Général de Gaulle Bât. P3, 4e étage, F-94010 Créteil Cedex, France. E-mail : hadiji@u-pec.fr

${ }^{\dagger}$ E-mail : habib.yazidi@u-pec.fr 
study the existence of solutions to problem (1.1).

The main motivation to consider such problem is the study of conformal deformations of Riemannian manifolds with boundary, see [6], [13] and [14].

Problem(1.1) has a variational form. Then the eventual solutions correspond to the critical points of the energy functional.

The existence of a solution of (1.1) is closely related to $S$ (resp. $S_{1}$ ) which is the best Sobolev constant for the imbedding $H^{1}(\Omega)$ into $L^{2^{*}}(\Omega)$ (resp. for the imbedding $H^{1}(\Omega)$ into $\left.L^{2 *}(\partial \Omega)\right)$. As in [4] for the nonlinear Dirichlet problem with critical Sobolev exponent, we will fill out the sufficient conditions to find solutions for the problem in presence of a nonlinear Neumann boundary data with a critical nonlinearity. One of the difficulty of our problem, besides the fact that the associated functional does not satisfy the Palais-Smale compactness condition (PS), is that it possesses four levels of homogeneity.

Let us recall some works related to the problem (1.1). If $p \equiv 1$ and $u$ satisfies homogeneous Dirichlet condition, problem (1.1) has been treated in [4], where the authors obtained positive solutions with energy less than $\frac{1}{N} S^{\frac{N}{2}}$, see also [15] and [7]. In [20], the author gives a complete description of the energy levels $c$, associated to problem (1.1), on which $(P S)_{c}$ sequence is not compact. For the case $p \not \equiv 1, f(x, u)=\lambda u$ with homogeneous Dirichlet condition we refer the reader to $[16,17]$. For the homogeneous Neumann problem, in [8], the authors proved the existence of solution with energy less than $\frac{1}{2 N} S^{\frac{N}{2}}$.

The case $p \equiv 1 \equiv Q, \beta=0$ and $f(x, u)$ is a linear perturbation, has an extensive literature and the first existence results was treated in $[1,3,9,10]$. In this case the solutions are obtained as minimizers of the variational problem associated to (1.1) with energy less than $S_{1}$. If $\beta=0$ and $f(x, u)$ has an explicit form, problem (1.1) has been studied in $[21,22]$ and some existence results are obtained.

In [11], the authors were interested to the case $p \equiv 1, f(x, u)=0$ and the presence of two critical nonlinearities. They derived some existence results by the use of the concentration compactness principle see [18]. For another form of equation (1.1) with competing critical nonlinearities, see [19] and references therein.

In this paper we are concerned with the general case, more precisely, $p \not \equiv 1, Q \not \equiv 0$ and $f(x, u) \neq 0$. We assume that $f$ is a lower-order perturbation of $|u|^{2^{*}-1}$ and $f(x, 0)=0$. Let $p_{0}=\min _{x \in \bar{\Omega}} p(x)$ and $x_{0} \in \partial \Omega$ satisfy

$$
\frac{\left(Q\left(x_{0}\right)\right)^{N-2}}{p\left(x_{0}\right)}=\max _{x \in \partial \Omega} \frac{|Q(x)|^{N-2}}{p(x)} .
$$

We assume that

$$
\left|p(x)-p\left(x_{0}\right)\right|=o\left(\left|x-x_{0}\right|\right)
$$

and

$$
\left|Q(x)-Q\left(x_{0}\right)\right|=o\left(\left|x-x_{0}\right|\right)
$$


for $x$ near $x_{0}$.

Our first contribution to problem (1.1), in section 2, is an existence result for the case where $\beta=0$. The energy solutions which we find are under the level on which the (PS) condition failed. More precisely, we show existence of solutions with energy in ]0, $\frac{1}{2(N-1)} \frac{p\left(x_{0}\right)}{\left(Q\left(x_{0}\right)\right)^{N-2}} S_{1}^{N-1}[$.

Next, in section 3, we turn to the general case and look for solutions for problem (1.1) in the case of the presence of competing critical nonlinearities in the case $p\left(x_{0}\right)=p_{0}$.

The main difficulty of the problem in caused by the presence of two critical exponents and a general nonlinear perturbation. This fact causes the change in energy level for which the Palais Smale condition (PS) is not satisfied. In this paper, we determine explicitly the new energy level $M\left(S, S_{1}\right)$ defined by

$$
M\left(S, S_{1}\right)=\frac{\frac{p\left(x_{0}\right)}{\left(Q\left(x_{0}\right)\right)^{N-2}} S_{1}^{N-1} 2^{N-2}}{[1+\sqrt{1+4 E}]^{N-2}}\left[\frac{1}{N}-\frac{N-2}{N(N-1)} \frac{1}{1+\sqrt{1+4 E}}\right]
$$

where $E=\left(\frac{\frac{p\left(x_{0}\right)}{Q\left(x_{0}\right)^{N-2}} S_{1}^{N-1}}{\left(p_{0} S\right)^{\frac{N}{2}}}\right)^{\frac{2}{N-2}}$. We will show the existence of solution for (1.1) with energy in $] 0, M\left(S, S_{1}\right)[$.

Note that

$$
0<M\left(S, S_{1}\right)<\min \left\{\frac{1}{2(N-1)} \frac{p\left(x_{0}\right)}{\left(Q\left(x_{0}\right)\right)^{N-2}} S_{1}^{N-1}, \frac{1}{N}\left(p_{0} S\right)^{\frac{N}{2}}\right\}
$$

\section{Existence results for $\beta=0$}

We assume that $f(x, u)$ can be written as

$$
f(x, u)=a(x) u+g(x, u)
$$

with

$$
a(x) \in L^{\infty}(\Omega)
$$

there exists $2<\alpha \leq 2_{*}$ such that, for every $x \in \mathbb{R}^{N}$ and $u \in \mathbb{R}$,

$$
\alpha G(x, u) \leq u g(x, u) \text {, where } G(x, u)=\int_{0}^{u} g(x, t) d t,
$$$$
|g(x, u)|=o(|u|) \quad \text { as } \quad u \rightarrow 0, \quad \text { uniformly in } x,
$$$$
|g(x, u)|=O\left(|u|^{2_{*}-1}\right) \quad \text { as }|u| \rightarrow+\infty, \quad \text { uniformly in } x \text {. }
$$

or

$$
|g(x, u)|=O\left(|u|^{r-1}\right) \quad \text { as }|u| \rightarrow+\infty, \quad \text { uniformly in } x \text {, }
$$

where $r$ is such that $2_{*}<r<2^{*}$, 
Moreover, we assume that the first eigenvalue $\lambda_{1}(a)$ of the following problem is positive:

$$
\begin{cases}-\operatorname{div}(p(x) u)-a(x) u=\mu u & \text { in } \Omega \\ \frac{\partial u}{\partial \nu}=0 & \text { on } \partial \Omega\end{cases}
$$

That is,

$$
\lambda_{1}(a)=\inf _{u \in H^{1}(\Omega)}\left\{\int_{\Omega}|\nabla u|^{2}-a(x) u^{2} d x, \int_{\Omega} u^{2} d x=1\right\}>0 .
$$

Under assumption (2.7), it is easy to verify that $\|u\|=\left(\int_{\Omega}|\nabla u|^{2}-a(x) u^{2} d x\right)^{\frac{1}{2}}$ is a norm on $H^{1}(\Omega)$ equivalent to the usual norm $\|\cdot\|_{H^{1}}$.

Let

$$
\Phi(u)=\frac{1}{2} \int_{\Omega} p(x)|\nabla u|^{2} d x-\int_{\Omega} F(x, u) d x-\frac{1}{2_{*}} \int_{\partial \Omega} p(x) Q(x)|u|^{2 *} d s_{x}, u \in H^{1}(\Omega),
$$

where $F(x, u)=\int_{0}^{u} f(x, t) d t$ for $x \in \bar{\Omega}, u \in \mathbb{R}$. Our main result in this section is

\section{Theorem 2.1}

Assume (2.1)-(2.5) and (2.7) or (2.1)-(2.4) and (2.6)-(2.7). Moreover suppose that

$$
\text { there exists some } v_{0} \in H^{1}, v_{0} \geq 0 \text { on } \Omega, v_{0} \neq 0 \text { on } \partial \Omega \text {, such that }
$$

$$
\sup _{t \geq 0} \Phi\left(t v_{0}\right)<\frac{1}{2(N-1)} \frac{p\left(x_{0}\right)}{\left(Q\left(x_{0}\right)\right)^{N-2}} S_{1}^{N-1} \text {. }
$$

Then problem (1.1) possesses a solution.

Proof of Theorem 2.1

Let $s=2_{*}$ when $f$ satisfies (2.5) and $s=r$ when $f$ satisfies (2.6). By (2.4) we have, for any $\varepsilon>0$, there is a $\delta>0$ such that

$$
|g(x, u)| \leq \varepsilon|u| \text { for a.e } x \in \Omega \text {, and for all }|u| \leq \delta,
$$

thus, by (2.5) or (2.6), we obtain

$$
|g(x, u)| \leq \varepsilon|u|+C|u|^{s-1} \text { for a.e } x \in \Omega \text {, and for all } u \in \mathbb{R},
$$

and for some constant $C$ (depending on $\varepsilon$ ). Therefore, we have

$$
F(x, u) \leq \frac{1}{2} a(x) u^{2}+\frac{\varepsilon}{2} u^{2}+\frac{C}{s}|u|^{s} \quad \text { for a.e } x \in \Omega \text {, and for all } u \in \mathbb{R} .
$$

Hence we find, for all $u \in H^{1}(\Omega)$,

$\Phi(u) \geq \frac{1}{2} \int_{\Omega} p(x)|\nabla u|^{2} d x-\frac{1}{2} \int_{\Omega} a(x)|u|^{2} d x-\frac{\varepsilon}{2} \int_{\Omega}|u|^{2} d x-\frac{C}{s} \int_{\Omega}|u|^{s} d x-\frac{1}{2_{*}} \int_{\partial \Omega} p(x) Q(x)|u|^{2 *} d s_{x}$

Using (2.7) we easily see that, for $\varepsilon>0$ small enough, there exist constants $k>0$, $C_{1}>0$ and $C_{2}>0$ such that

$$
\begin{aligned}
\Phi(u) & \geq k\|u\|^{2}-C_{1}\|u\|^{s}-C_{2}\|u\|^{2_{*}} \\
& \geq\|u\|^{2}\left(k-C_{1}\|u\|^{s-2}-C_{2}\|u\|^{2_{*}-2}\right) \quad \text { for all } u \in H^{1},
\end{aligned}
$$


which implies, since $2_{*}>2$ and $s>2$, for some small $\alpha>0$ there exists $\rho>0$ such that

$$
\Phi(u) \geq \rho, \quad \text { provided }\|u\|=\alpha .
$$

At this stage, we need some notations and some estimations. We recall $S_{1}$ defined by

$$
S_{1}=\inf \left\{\int_{\mathbb{R}_{+}^{N}}|\nabla u|^{2} d x ; u \in H^{1}\left(\mathbb{R}_{+}^{N}\right), \int_{\mathbb{R}^{N-1}}|u|^{2 *} d x=1\right\}
$$

the best constant for the trace embedding $H^{1}\left(\mathbb{R}_{+}^{N}\right)$ into $L^{q}\left(\partial \mathbb{R}_{+}^{N}\right)$, where $R_{+}^{N}=\{x=$ $\left.\left(x^{\prime}, x_{N}\right): x^{\prime} \in \mathbb{R}^{N-1}, x_{N}>0\right\}$.

We recall from [13] and [18] that the minimizing functions of $S_{1}$ are of the form

$$
W(x)=\frac{\gamma_{N}}{\left[\left|x^{\prime}\right|^{2}+\left(1+x_{N}\right)^{2}\right]^{\frac{N-2}{2}}}
$$

where $\gamma_{N}$ is a positive constant depending on $N$. We set

$$
W_{\varepsilon, x_{0}}(x)=\varepsilon^{-\frac{N-2}{2}} \phi(x) W\left(\frac{x-x_{0}}{\varepsilon}\right),
$$

where $x_{0} \in \partial \Omega$ and $\phi$ is a radial $C^{\infty}$-function such that

$$
\phi(x)= \begin{cases}1 & \text { if }\left|x-x_{0}\right| \leq \frac{R}{4} \\ 0 & \text { if }\left|x-x_{0}\right|>\frac{R}{2}\end{cases}
$$

with $R>0$ is a small constant.

From [3] and [10] we have the following estimates

$$
\int_{\Omega} p(x)\left|\nabla W_{\varepsilon, x_{0}}\right|^{2} d x=p\left(x_{0}\right) A_{1}-p\left(x_{0}\right) H\left(x_{0}\right) \begin{cases}A_{2}^{\prime} \varepsilon|\log \varepsilon|+o(\varepsilon|\log \varepsilon|) & \text { if } N=3 \\ A_{2} \varepsilon+o(\varepsilon) & \text { if } N \geq 4,\end{cases}
$$

$$
\int_{\partial \Omega} p(x) Q(x)\left|W_{\varepsilon, x_{0}}\right|^{2 *} d s_{x}=p\left(x_{0}\right) Q\left(x_{0}\right)\left(B_{1}-H\left(x_{0}\right) B_{2} \varepsilon\right)+o(\varepsilon)
$$

where $A_{1}, A_{2}^{\prime}, A_{2}, B_{1}$ and $B_{2}$ are some positive constants defined explicitly in [3]. From [21], for some $2<r<2^{*}$, we have

$$
\int_{\Omega}\left|W_{\varepsilon, x_{0}}\right|^{r} d x= \begin{cases}o(\varepsilon) & \text { if } N \geq 4 \\ o(\varepsilon|\ln (\varepsilon)|) & \text { If } N=3 .\end{cases}
$$

Let us notice that

$$
S_{1}=\frac{A_{1}}{B_{1}^{\frac{2}{2 *}}} \quad \text { and } \quad A_{2}-\frac{2}{2_{*}} \frac{A_{1} B_{2}}{B_{1}}>0
$$

On the other hand, when $f$ satisfies (2.5), we easily see that $\lim _{t \rightarrow+\infty} \Phi\left(t W_{\varepsilon, x_{0}}\right)=-\infty$. Then we take $v=t_{0} W_{\varepsilon, x_{0}}$, where $t_{0}>0$ is chosen large enough so that $\|v\|>\alpha$ and 
$\Phi(v) \leq 0$.

When $f$ satisfies (2.6), using (2.12)-(2.14), we have

$$
\Phi\left(t W_{\varepsilon, x_{0}}\right)=t^{2} A-t^{2 *} B+t^{r} \begin{cases}o(\varepsilon) & \text { if } N \geq 4 \\ o(\varepsilon|\ln (\varepsilon)|) & \text { if } N=3 .\end{cases}
$$

Therefore, for $\varepsilon>0$ small enough, there exists many $t_{0}>0$ such that $t_{0}^{2} A-t_{0}^{2 *} B<0$. Let, again, $v=t_{0} W_{\varepsilon, x_{0}}$ for $\varepsilon$ small enough when $t_{0}$ is chosen large such that $\|v\|>\alpha$ and $\Phi(v) \leq 0$.

Set

$$
c=\inf _{\mathcal{P} \in \mathcal{A}} \max _{w \in \mathcal{P}} \Phi(w),
$$

where $\mathcal{A}$ denotes the class of continuous paths joining 0 to $v$.

Thanks to a result of Ambrosetti and Rabinowtz [2], see also [4], there exists a sequence $\left\{u_{j}\right\}$ in $H^{1}(\Omega)$ such that

$$
\Phi\left(u_{j}\right) \rightarrow c \quad \text { and } \quad \Phi^{\prime}\left(u_{j}\right) \rightarrow 0 \text { in } H^{-1}(\Omega) .
$$

Looking at $(2.8)$ we see that $c<\frac{1}{2(N-1)} \frac{p\left(x_{0}\right)}{\left(Q\left(x_{0}\right)\right)^{N-2}} S_{1}^{N-1}$.

In order to conclude the proof of Theorem 2.1, we need the following Lemma.

\section{Lemma 2.1}

Let $\left\{u_{j}\right\} \subset H^{1}(\Omega)$ be a sequence satisfying

$$
\Phi\left(u_{j}\right) \rightarrow c<\frac{p\left(x_{0}\right) S_{1}^{N-1}}{2(N-1)\left(Q\left(x_{0}\right)\right)^{N-2}}
$$

and

$$
\Phi^{\prime}\left(u_{j}\right) \rightarrow 0 \quad \text { in } H^{-1}(\Omega)
$$

then $\left\{u_{j}\right\}$ is relatively compact in $H^{1}(\Omega)$.

\section{Proof of Lemma 2.1:}

We start by showing that $\left\{u_{j}\right\}$ is bounded in $H^{1}(\Omega)$.

Using (2.1) and (2.7) we see that (2.17) and (2.18) are equivalent to

$$
\frac{1}{2}\left\|u_{j}\right\|^{2}-\int_{\Omega} G\left(x, u_{j}\right) d x-\frac{1}{2_{*}} \int_{\partial \Omega} p(x) Q(x)\left|u_{j}\right|^{2 *} d s_{x}=c+o(1),
$$

and

$$
\left\|u_{j}\right\|^{2}-\int_{\Omega} g\left(x, u_{j}\right) u_{j} d x-\int_{\partial \Omega} p(x) Q(x)\left|u_{j}\right|^{2 *} d s_{x}=<\xi_{j}, u_{j}>
$$

with $\xi_{j} \rightarrow 0$ in $H^{-1}$.

Taking (2.19)- $\frac{1}{2}(2.20)$, we get

$$
\frac{1}{2(N-1)} \int_{\partial \Omega} p(x) Q(x)\left|u_{j}\right|^{2 *} d s_{x}-\int_{\Omega} G\left(x, u_{j}\right) d x+\frac{1}{2} \int_{\Omega} g\left(x, u_{j}\right) u_{j} d x=c+o\left(\left\|u_{j}\right\|\right) .
$$


On the other hand, (2.19)-- $\frac{1}{2 *}(2.20)$ yields

$$
\frac{1}{2(N-1)}\left\|u_{j}\right\|^{2}-\int_{\Omega} G\left(x, u_{j}\right) d x+\frac{1}{2_{*}} \int_{\Omega} g\left(x, u_{j}\right) u_{j} d x=c+o\left(\left\|u_{j}\right\|\right) .
$$

Using (2.3), (2.21) and (2.22) follow

$$
\frac{1}{2(N-1)}\left\|u_{j}\right\|^{2}-\left(1-\frac{\alpha}{2_{*}}\right) \int_{\Omega} G\left(x, u_{j}\right) d x \leq c+o\left(\left\|u_{j}\right\|\right)
$$

and

$$
\frac{1}{2(N-1)} \int_{\partial \Omega} p(x) Q(x)\left|u_{j}\right|^{2 *} d s_{x}-\left(1-\frac{\alpha}{2}\right) \int_{\Omega} G\left(x, u_{j}\right) d x \leq c+o\left(\left\|u_{j}\right\|\right) .
$$

Computing $\left(\frac{\alpha}{2}-1\right)(2.23)+\left(1-\frac{\alpha}{2_{*}}\right)(2.24)$, we obtain

$$
\left(\frac{\alpha}{2}-1\right)\left\|u_{j}\right\|^{2}+\left(1-\frac{\alpha}{2_{*}}\right) \int_{\partial \Omega} p(x) Q(x)\left|u_{j}\right|^{2 *} d s_{x} \leq c+o\left(\left\|u_{j}\right\|\right) .
$$

Therefore, since $2<\alpha \leq 2_{*}$, we obtain that $\left\{u_{j}\right\}$ is bounded in $H^{1}(\Omega)$.

Extract a subsequence, still denoted by $u_{j}$, such that

$$
\begin{aligned}
u_{j} & \rightarrow u \quad \text { weakly in } H^{1}(\Omega), \\
u_{j} & \rightarrow u \quad \text { strongly in } L^{t}(\Omega) \text { for all } t<2^{*}=\frac{2 N}{N-2}, \\
u_{j} & \rightarrow u \quad \text { a.e. on } \Omega, \\
f\left(x, u_{j}\right) & \rightarrow f(x, u) \quad \text { strongly in } L^{\frac{r}{r-1}}(\Omega), \\
u_{j} & \rightarrow u \quad \text { weakly in } L^{2_{*}}(\partial \Omega) .
\end{aligned}
$$

Passing to the limit in (2.18), we obtain

$$
\begin{cases}-\operatorname{div}(p(x) \nabla u)=f(x, u) & \text { in } \Omega \\ \frac{\partial u}{\partial \nu}=Q(x)|u|^{2 *}-2 & \text { on } \partial \Omega\end{cases}
$$

We shall now verify that $u \not \equiv 0$. Indeed, suppose that $u \equiv 0$. We claim that

$$
\int_{\Omega} f\left(x, u_{j}\right) u_{j} d x \rightarrow 0 \text { and } \int_{\Omega} F\left(x, u_{j}\right) d x \rightarrow 0 .
$$

From (2.5) or (2.6), let $s=2_{*}$ if $f$ satisfies (2.5) and $s=r$ if $f$ satisfies (2.6), we have

$$
\begin{aligned}
& \text { for some constants } C_{1}>0 \text { and } C_{2}>0 \\
& |f(x, u)| \leq C_{1}|u|^{s-1}+C_{2} \quad \text { for a.e. } x \in \Omega, \text { and for all } u \in \mathbb{R},
\end{aligned}
$$

and then

$$
|F(x, u)| \leq \frac{C_{1}}{s}|u|^{s}+C_{2}|u| \text { for a.e } x \in \Omega \text {, and for all } u \in \mathbb{R} \text {. }
$$

Therefore

$$
\left|\int_{\Omega} f\left(x, u_{j}\right) u_{j} d x\right| \leq C_{1} \int_{\Omega}\left|u_{j}\right|^{s} d x+C_{2} \int_{\Omega}\left|u_{j}\right| d x
$$


and

$$
\left|\int_{\Omega} F\left(x, u_{j}\right) d x\right| \leq \frac{C_{1}}{s} \int_{\Omega}\left|u_{j}\right|^{s} d x+C_{2} \int_{\Omega}\left|u_{j}\right| d x .
$$

Since $u_{j} \rightarrow 0$ in $L^{s}(\Omega)$ then for $j$ large enough, we have

$$
\int_{\Omega} f\left(x, u_{j}\right) u_{j} d x=o(1)
$$

and

$$
\int_{\Omega} F\left(x, u_{j}\right) d x=o(1) .
$$

Which gives the desired result.

Extracting a subsequence, still denoted by $u_{j}$, we may assume that

$$
\int_{\Omega} p(x)\left|\nabla u_{j}\right|^{2} d x \rightarrow l \quad \text { for some constant } l \geq 0 .
$$

Passing to the limit in (2.20), we obtain

$$
\int_{\partial \Omega} p(x) Q(x)\left|u_{j}\right|^{2_{*}} d s_{x} \rightarrow l .
$$

Passing to the limit in (2.21), we easily get

$$
\frac{1}{2(N-1)} l=c
$$

Therefore $l>0$ and $\int_{\partial \Omega} p(x) Q(x)\left|u_{j}\right|^{2 *} d s_{x}>0$ for large $j$.

On the other hand, from the result of [24, Theorem 02], we know that there exists a constant $C(\Omega)>0$ such that for every $w \in H^{1}(\Omega)$

$$
\int_{\Omega}|\nabla w|^{2} d x+C(\Omega) \int_{\Omega}|w|^{k} d x \geq S_{1}\left(\int_{\partial \Omega}|w|^{2_{*}} d s_{x}\right)^{\frac{2}{2_{*}}}
$$

with $k=\frac{2 N}{N-1}$ if $N \geq 4$ and $k>3=\frac{2 N}{N-1}$ if $N=3$.

We apply this result for $w_{j}=(p(x))^{\frac{1}{2}} u_{j}$ and in particular for $N=3$ we take $k$ such that $6=\frac{2 N}{N-2}>k>3$, we obtain for $j$ large enough

$$
\int_{\Omega}\left|\nabla(p(x))^{\frac{1}{2}} u_{j}\right|^{2} d x+C(\Omega) \int_{\Omega}\left|(p(x))^{\frac{1}{2}} u_{j}\right|^{k} d x \geq S_{1}\left(\int_{\partial \Omega}\left|(p(x))^{\frac{1}{2}} u_{j}\right|^{2_{*}} d s_{x}\right)^{\frac{2}{2_{*}}}
$$

Since $k<\frac{2 N}{N-2}$ for every $N \geq 3$, thanks to the compact embedding $H^{1}(\Omega) \hookrightarrow L^{k}(\Omega)$, we have, for a subsequence, $u_{j} \rightarrow 0$ strongly in $L^{k}(\Omega)$ and we deduce

$$
\int_{\Omega} p(x)\left|\nabla u_{j}\right|^{2} d x+o(1) \geq S_{1}\left(\int_{\partial \Omega}\left|(p(x))^{\frac{1}{2}} u_{j}\right|^{2 *} d s_{x}\right)^{\frac{2}{2 *}}+o(1) .
$$

Using the fact that

$$
\frac{|Q(x)|^{N-2}}{p(x)} \leq \frac{\left(Q\left(x_{0}\right)\right)^{N-2}}{p\left(x_{0}\right)} \quad \forall x \in \partial \Omega
$$


(2.28) becomes

$$
\begin{aligned}
\int_{\Omega} p(x)\left|\nabla u_{j}\right|^{2} d x+o(1) & \geq S_{1}\left(\int_{\partial \Omega}(p(x))^{\frac{2 *}{2}}\left(\frac{\frac{|Q(x)|^{N-2}}{p(x)}}{\frac{\left(Q\left(x_{0}\right)\right)^{N-2}}{p\left(x_{0}\right)}}\right)^{\frac{1}{N-2}}\left|u_{j}\right|^{2 *} d s_{x}\right)^{\frac{2}{2 *}}+o(1) \\
& \geq S_{1}\left[\frac{\left(p\left(x_{0}\right)\right)^{\frac{1}{N-2}}}{Q\left(x_{0}\right)}\right]^{\frac{2}{2 *}}\left(\int_{\partial \Omega} p(x)|Q(x)|\left|u_{j}\right|^{2 *} d s_{x}\right)^{\frac{2}{2 *}}+o(1) \\
& \geq S_{1}\left[\frac{\left(p\left(x_{0}\right)\right)^{\frac{1}{N-2}}}{Q\left(x_{0}\right)}\right]^{\frac{2}{2 *}}\left(\int_{\partial \Omega} p(x) Q(x)\left|u_{j}\right|^{2_{*}} d s_{x}\right)^{\frac{2}{2 *}}+o(1) .
\end{aligned}
$$

At the limit we obtain

$$
l \geq\left[\frac{\left(p\left(x_{0}\right)\right)^{\frac{1}{N-2}}}{Q\left(x_{0}\right)}\right]^{\frac{2}{2 *}} S_{1} l^{\frac{N-2}{N-1}}
$$

and

$$
l \geq \frac{\left(p\left(x_{0}\right)\right)^{\frac{1}{N-1}}}{\left(Q\left(x_{0}\right)\right)^{\frac{N-2}{N-1}}} S_{1} l^{\frac{N-2}{N-1}} .
$$

Using (3.9) and (2.27) we see that $l \not \equiv 0$ and

$$
l^{\frac{1}{N-1}} \geq \frac{\left(p\left(x_{0}\right)\right)^{\frac{1}{N-1}}}{\left(Q\left(x_{0}\right)\right)^{\frac{N-2}{N-1}}} S_{1} .
$$

Therefore

$$
l \geq \frac{p\left(x_{0}\right)}{\left(Q\left(x_{0}\right)\right)^{N-2}} S_{1}^{N-1}
$$

and from (2.27) we have

$$
c \geq \frac{1}{2(N-1)} \frac{p\left(x_{0}\right)}{\left(Q\left(x_{0}\right)\right)^{N-2}} S_{1}^{N-1}
$$

which gives a contradiction with the fact that $c<\frac{1}{2(N-1)} \frac{p\left(x_{0}\right)}{\left(Q\left(x_{0}\right)\right)^{N-2}} S_{1}^{N-1}$, thus $u \not \equiv 0$. Now, we shall prove, for a subsequence, that $u_{j} \rightarrow u$ strongly in $H^{1}(\Omega)$.

We start by showing that $\Phi(u) \geq 0$. Indeed, since $u$ is a solution of (1.1) with $\beta=0$, we have

$$
\int_{\Omega} p(x)|\nabla u|^{2} d x=\int_{\Omega} f(x, u) d x+\int_{\partial \Omega} p(x) Q(x)|u|^{2_{*}} d s_{x} .
$$

On the other hand

$$
\Phi(u)=\frac{1}{2} \int_{\Omega} p(x)|\nabla u|^{2} d x-\frac{1}{2_{*}} \int_{\partial \Omega} p(x) Q(x)|u|^{2 *} d s_{x}-\int_{\Omega} F(x, u) d x .
$$

Therefore, using (2.3), we have

$$
\Phi(u) \geq \frac{1}{2(N-1)} \int_{\Omega} p(x)|\nabla u|^{2} d x+\left(\frac{\alpha}{2_{*}}-1\right) \int_{\Omega} F(x, u) d x,
$$

and

$$
\Phi(u) \geq \frac{1}{2(N-1)} \int_{\partial \Omega} p(x) Q(x)|u|^{2 *} d s_{x}+\left(\frac{\alpha}{2}-1\right) \int_{\Omega} F(x, u) d x .
$$


Since $2<\alpha \leq 2_{*}$, we deduce that $\phi(u) \geq 0$.

We set $v_{j}=u_{j}-u$. We have

$$
\int_{\Omega} p(x)\left|\nabla u_{j}\right|^{2} d x=\int_{\Omega} p(x)|\nabla u|^{2} d x+\int_{\Omega} p(x)\left|\nabla v_{j}\right|^{2} d x+o(1)
$$

and from [5] we deduce that

$$
\int_{\partial \Omega} p(x) Q(x)\left|u_{j}\right|^{2 *} d s_{x}=\int_{\partial \Omega} p(x) Q(x)|u|^{2 *} d s_{x}+\int_{\partial \Omega} p(x) Q(x)\left|v_{j}\right|^{2 *} d s_{x}+o(1) .
$$

Inserting (2.30) and (2.31) into (2.19) and (2.20) we get

$$
\Phi(u)+\frac{1}{2} \int_{\Omega} p(x)\left|\nabla v_{j}\right|^{2} d x-\frac{1}{2_{*}} \int_{\partial \Omega} p(x) Q(x)\left|v_{j}\right|^{2 *} d s_{x}=c+o(1)
$$

and (looking at (2.18))

$$
\int_{\Omega} p(x)\left|\nabla v_{j}\right|^{2} d x-\int_{\partial \Omega} p(x) Q(x)\left|v_{j}\right|^{2_{*}} d s_{x}=o(1) .
$$

Extracting a subsequence, still denoted by $u_{j}$, we may assume that

$$
\int_{\Omega} p(x)\left|\nabla v_{j}\right|^{2} d x \rightarrow l \quad \text { for some constant } l \geq 0
$$

From (2.33) we obtain

$$
\int_{\partial \Omega} p(x) Q(x)\left|v_{j}\right|^{2} d s_{x}=l .
$$

Passing to the limit in (2.32), we easily see that

$$
\frac{1}{2(N-1)} l=c-\Phi(u)
$$

Using the Sobolev embedding, see (2.29) for details, we have

$$
l \geq \frac{\left(p\left(x_{0}\right)\right)^{\frac{1}{N-2}}}{\left(Q\left(x_{0}\right)\right)^{\frac{N-2}{N-1}}} S_{1} l^{\frac{N-2}{N-1}} .
$$

We claim that $l=0$. Indeed, arguing by contradiction, assuming that $l \neq 0$, then $(2.35)$ gives

$$
l \geq \frac{p\left(x_{0}\right)}{\left(Q\left(x_{0}\right)\right)^{N-2}} S_{1}^{N-1}
$$

From (2.34), we obtain

$$
c-\Phi(u) \geq \frac{1}{2(N-1)} \frac{p\left(x_{0}\right)}{\left(Q\left(x_{0}\right)\right)^{N-2}} S_{1}^{N-1}
$$

which gives a contradiction, since $c<\frac{1}{2(N-1)} \frac{\left(p\left(x_{0}\right)\right.}{\left(Q\left(x_{0}\right)\right)^{N-2}} S_{1}^{N-1}$ and $\Phi(u) \geq 0$. Therefore $l=0, c=\Phi(u)$ and $u_{j} \rightarrow u$ strongly in $H^{1}(\Omega)$. 


\subsection{Sufficient conditions on $f(x, u)$ which give condition (2.8):}

We claim that $W_{\varepsilon, x_{0}}$ satisfies condition (2.8) for $\varepsilon>0$ sufficiently small. Indeed, we have $\Phi\left(t W_{\varepsilon, x_{0}}\right)=\frac{1}{2} t^{2} \int_{\Omega} p(x)\left|\nabla W_{\varepsilon, x_{0}}\right|^{2} d x-\frac{t^{2 *}}{2_{*}} \int_{\partial \Omega} p(x) Q(x)\left|W_{\varepsilon, x_{0}}\right|^{2 *} d s_{x}-\int_{\Omega} F\left(x, t W_{\varepsilon, x_{0}}\right) d x$.

When $f$ satisfies $(2.5)$, we easily see that $\lim _{t \rightarrow+\infty} \Phi\left(t W_{\varepsilon, x_{0}}\right)=-\infty$ and for large $t_{0}>0$ we have $\Phi\left(t_{0} W_{\varepsilon, x_{0}}\right)<0$.

When $f$ satisfies (2.6), using (2.12), (2.13) and (2.14), we have

$$
\Phi\left(t W_{\varepsilon, x_{0}}\right)=t^{2} A-t^{2 *} B+t^{r} \begin{cases}o(\varepsilon) & \text { if } N \geq 4 \\ o(\varepsilon|\ln (\varepsilon)|) & \text { if } N=3 .\end{cases}
$$

Therefore, for $\varepsilon>0$ small enough, we chose $t_{0}>0$ such that $t_{0}^{2} A-t_{0}^{2 *} B<0$ and $\Phi\left(t_{0} W_{\varepsilon, x_{0}}\right)<0$. Therefore, in both cases, $\sup _{t \in[0,1]} \Phi\left(t t_{0} W_{\varepsilon, x_{0}}\right)$ is achieved at some $0 \leq \tilde{t}_{\varepsilon} \leq 1$ and $\tilde{t}_{\varepsilon}$ is bounded. In the rest of this section, we note $t_{\varepsilon}=\tilde{t}_{\varepsilon} t_{0}$.

From now, we can suppose that $t_{\varepsilon}>0$, indeed if $t_{\varepsilon}=0$ then $\sup _{t \geq 0} \Phi\left(t W_{\varepsilon, x_{0}}\right)=0$ and the condition (2.8) is satisfied.

Since the derivative of the function $t \rightarrow \Phi\left(t W_{\varepsilon, x_{0}}\right)$ vanishes at $t_{\varepsilon}$ we have

$$
t_{\varepsilon} \int_{\Omega} p(x)\left|\nabla W_{\varepsilon, x_{0}}\right|^{2} d x-t_{\varepsilon}^{2_{*}-1} \int_{\partial \Omega} p(x) Q(x)\left|W_{\varepsilon, x_{0}}\right|^{2 *} d s_{x}-\int_{\Omega} f\left(x, t_{\varepsilon} W_{\varepsilon, x_{0}}\right) W_{\varepsilon, x_{0}} d x=0 .
$$

We claim that

$$
\int_{\Omega} \frac{f\left(x, t_{\varepsilon} W_{\varepsilon, x_{0}}\right) W_{\varepsilon, x_{0}}}{t_{\varepsilon}} d x \rightarrow 0 \text { as } \varepsilon \rightarrow 0 .
$$

Indeed, from (2.36), we have

$$
\int_{\Omega} p(x)\left|\nabla W_{\varepsilon, x_{0}}\right|^{2} d x-t_{\varepsilon}^{2_{*}-2} \int_{\partial \Omega} p(x) Q(x)\left|W_{\varepsilon, x_{0}}\right|^{2 *} d s_{x}-\int_{\Omega} \frac{f\left(x, t_{\varepsilon} W_{\varepsilon, x_{0}}\right) W_{\varepsilon, x_{0}}}{t_{\varepsilon}} d x=0 .
$$

Using (2.1)-(2.5) or (2.1)-(2.4) and (2.6), there are $C_{1}>0$ and $C_{2}>0$ such that, for a.e. $x \in \Omega$, for all $u \in \mathbb{R},|f(x, u)| \leq C_{1}|u|^{s-1}+C_{2}|u|$ where $s=2_{*}$ if $f$ satisfies (2.5) and $s=r$ if $f$ satisfies (2.6).

Therefore

$$
\int_{\Omega} \frac{f\left(x, t_{\varepsilon} W_{\varepsilon, x_{0}}\right) W_{\varepsilon, x_{0}}}{t_{\varepsilon}} d x \leq C_{1} t_{\varepsilon}{ }^{s-2}\left\|W_{\varepsilon, x_{0}}\right\|_{L^{s}}^{s}+C_{2}\left\|W_{\varepsilon, x_{0}}\right\|_{L^{2}}^{2},
$$

Using the fact that, as $\varepsilon \rightarrow 0, t_{\varepsilon}$ is bounded, $\left\|W_{\varepsilon, x_{0}}\right\|_{L^{2}(\Omega)} \rightarrow 0$ and $\left\|W_{\varepsilon, x_{0}}\right\|_{L^{s}(\Omega)} \rightarrow 0$ since $s<2^{*}$, we get directly $(2.37)$.

Consequently, for $\varepsilon>0$ small enough, (2.36) become

$$
t_{\varepsilon} \int_{\Omega} p(x)\left|\nabla W_{\varepsilon, x_{0}}\right|^{2} d x-t_{\varepsilon}^{2_{*}-1} \int_{\partial \Omega} p(x) Q(x)\left|W_{\varepsilon, x_{0}}\right|^{2 *} d s_{x}=o(1) .
$$

Therefore

$$
t_{\varepsilon} \leq\left(\frac{\int_{\Omega} p(x)\left|\nabla W_{\varepsilon, x_{0}}\right|^{2} d x}{\int_{\partial \Omega} p(x) Q(x)\left|W_{\varepsilon, x_{0}}\right|^{2 *} d s_{x}}\right)^{\frac{1}{2 *-2}}+o(1) .
$$


Set

$$
X_{\varepsilon}=\left(\frac{\int_{\Omega} p(x)\left|\nabla W_{\varepsilon, x_{0}}\right|^{2} d x}{\int_{\partial \Omega} p(x) Q(x)\left|W_{\varepsilon, x_{0}}\right|^{2 *} d s_{x}}\right)^{\frac{1}{2 *-2}}
$$

and

$$
M_{\varepsilon}=\sup _{t \in[0,1]} \Phi\left(t t_{0} W_{\varepsilon, x_{0}}\right)=\Phi\left(t_{\varepsilon} W_{\varepsilon, x_{0}}\right) .
$$

Since the function $t \rightarrow \frac{1}{2} t^{2} \int_{\Omega} p(x)\left|\nabla W_{\varepsilon, x_{0}}\right|^{2} d x-\frac{t^{2 *}}{2_{*}} \int_{\partial \Omega} p(x) Q(x)\left|W_{\varepsilon, x_{0}}\right|^{2 *} d s_{x}$ is increasing on the interval $\left[0, X_{\varepsilon}\right]$ we have, by $(2.38)$,

$$
M_{\varepsilon} \leq \frac{1}{2} X_{\varepsilon}^{2} \int_{\Omega} p(x)\left|\nabla W_{\varepsilon, x_{0}}\right|^{2} d x-\frac{X_{\varepsilon}^{2 *}}{2_{*}} \int_{\partial \Omega} p(x) Q(x)\left|W_{\varepsilon, x_{0}}\right|^{2 *} d s_{x}-\int_{\Omega} F\left(x, t_{\varepsilon} W_{\varepsilon, x_{0}}\right) d x .
$$

Using (2.12)-(2.15) and the fact that $\int_{\Omega}\left|W_{\varepsilon, x_{0}}\right|^{2} d x=o(\varepsilon)$, we obtain

$$
\begin{aligned}
M_{\varepsilon} \leq & \frac{1}{2(N-1)} \frac{p\left(x_{0}\right)}{\left(Q\left(x_{0}\right)\right)^{N-2}} S_{1}^{N-1}-\int_{\Omega} G\left(x, t_{\varepsilon} W_{\varepsilon, x_{0}}\right) d x \\
& - \begin{cases}H\left(x_{0}\right)\left(\frac{A_{1}}{Q\left(x_{0}\right) B_{1}}\right)^{\frac{2}{2 *-2}} A_{2}^{\prime} \varepsilon|\log \varepsilon|+o(\varepsilon|\log \varepsilon|) & \text { if } N=3, \\
H\left(x_{0}\right) \frac{p\left(x_{0}\right)}{2}\left(A_{2}-\frac{2}{2_{*}} \frac{A_{1} B_{2}}{B_{1}}\right) \varepsilon+o(\varepsilon) & \text { if } N \geq 4 .\end{cases}
\end{aligned}
$$

At this stage, we distinguish two cases:

When $H\left(x_{0}\right) \leq 0$.

\section{Lemma 2.2}

Assume that $f(x, u)$ satisfies (2.1)-(2.5) and (2.7) or (2.1)-(2.4) and (2.6)-(2.7). Suppose that there exists some continuous function $g($.$) such that$

$$
g(x, u) \geq g(u) \text { for a.e. } x \in \Omega \text { and for all } u \in \mathbb{R}
$$

and the primitive $G(u)=\int_{0}^{u} g(t) d t$ satisfies, for $N \geq 4$

$$
\lim _{\varepsilon \rightarrow 0} \varepsilon^{\frac{N-2}{2}} \int_{\varepsilon^{\frac{1}{2}}}^{+\infty} t^{N-1} \int_{0}^{+\infty} G\left(\frac{t^{-(N-2)}}{\left(1+r^{2}\right)^{\frac{(N-2)}{2}}}\right) r^{N-2} d r d t=+\infty .
$$

and for $N=3$

$$
\lim _{\varepsilon \rightarrow 0} \frac{\varepsilon^{\frac{1}{2}}}{|\ln (\varepsilon)|} \int_{\varepsilon^{\frac{1}{2}}}^{+\infty} t^{2} \int_{0}^{+\infty} G\left(\frac{t^{-1}}{\left(1+r^{2}\right)^{\frac{1}{2}}}\right) r d r d t=+\infty .
$$

Then condition (2.8) holds.

Proof.

From (2.40) and (2.11), for $\varepsilon>0$ sufficiently small, we have

$$
\int_{\Omega} G\left(x, t_{\varepsilon} W_{\varepsilon, x_{0}}\right) d x \geq \int_{\Omega} G\left(\frac{A \varepsilon^{\frac{N-2}{2}}}{\left[\left(\varepsilon+x_{N}\right)^{2}+\left|x^{\prime}-x_{0}^{\prime}\right|^{2}\right]^{\frac{N-2}{2}}}\right) d x
$$


for some constant $A>0$.

Inserting (2.43) into (2.39) we write

$$
\begin{aligned}
M \varepsilon & \leq \frac{1}{2(N-1)} \frac{p\left(x_{0}\right)}{\left(Q\left(x_{0}\right)\right)^{N-2}} S_{1}^{N-1}-\int_{\Omega} G\left(\frac{A \varepsilon^{\frac{N-2}{2}}}{\left[\left(\varepsilon+\left(x_{N}-x_{0 N}\right)\right)^{2}+\left|x^{\prime}-x_{0}^{\prime}\right|^{2}\right]^{\frac{N-2}{2}}}\right) d x \\
& - \begin{cases}H\left(x_{0}\right)\left(\frac{A_{1}}{Q\left(x_{0}\right) B_{1}}\right)^{\frac{2}{2 *-2}} A_{2}^{\prime} \varepsilon|\log \varepsilon|+o(\varepsilon|\log \varepsilon|) & \text { if } N=3, \\
H\left(x_{0}\right) \frac{p\left(x_{0}\right)}{2}\left(A_{2}-\frac{2}{2_{*}} \frac{A_{1} B_{2}}{B_{1}}\right) \varepsilon+o(\varepsilon) & \text { if } N \geq 4 .\end{cases}
\end{aligned}
$$

Finally, we claim that

$$
\lim _{\varepsilon \rightarrow 0} \frac{1}{\varepsilon} \int_{\Omega} G\left(\frac{A \varepsilon^{\frac{N-2}{2}}}{\left[\left(\varepsilon+x_{N}\right)^{2}+\left|x^{\prime}-x_{0}^{\prime}\right|^{2}\right]^{\frac{N-2}{2}}}\right) d x=+\infty \text { If } N \geq 4
$$

and

$$
\lim _{\varepsilon \rightarrow 0} \frac{1}{\varepsilon|\log \varepsilon|} \int_{\Omega} G\left(\frac{A \varepsilon^{\frac{1}{2}}}{\left[\left(\varepsilon+x_{N}\right)^{2}+\left|x^{\prime}-x_{0}^{\prime}\right|^{2}\right]^{\frac{N-2}{2}}}\right) d x=+\infty \text { If } N=3
$$

which implies, together with $(2.44)$, that $M_{\varepsilon}<\frac{1}{2(N-1)} \frac{p\left(x_{0}\right)}{\left(Q\left(x_{0}\right)\right)^{N-2}} S_{1}^{N-1}$ for $\varepsilon>0$ sufficiently small.

Verification of (2.45) and (2.46):

$$
\begin{array}{r}
\int_{\Omega} G\left(\frac{A \varepsilon^{\frac{N-2}{2}}}{\left[\left(\varepsilon+x_{N}\right)^{2}+\left|x^{\prime}-x_{0}^{\prime}\right|^{2}\right]^{\frac{N-2}{2}}}\right) d x=\varepsilon^{N} \int_{\mathbb{R}_{+}^{N}} G\left(\frac{A \varepsilon^{-\frac{N-2}{2}}}{\left[\left(1+y_{N}\right)^{2}+\left|y^{\prime}\right|^{2}\right]^{\frac{N-2}{2}}}\right) d y+O(1) \\
=\varepsilon^{N} \omega \int_{0}^{+\infty} \int_{0}^{+\infty}\left(1+y_{N}\right)^{N-1} G\left(\frac{1}{\left(1+y_{N}\right)^{N-2}} \frac{A \varepsilon^{-\frac{N-2}{2}}}{\left[1+r^{2}\right]^{\frac{N-2}{2}}}\right) r^{N-2} d r d y_{N}
\end{array}
$$

where $\omega$ is the area of sphere $S^{N-2}$.

Using the change of variable $t=\varepsilon^{\frac{1}{2}}\left(1+y_{N}\right)$ we get

$$
\int_{\Omega} G\left(\frac{A \varepsilon^{\frac{N-2}{2}}}{\left[\left(\varepsilon+x_{N}\right)^{2}+\left|x^{\prime}-x_{0}^{\prime}\right|^{2}\right]^{\frac{N-2}{2}}}\right) d x=\varepsilon^{\frac{N}{2}} \omega \int_{\varepsilon^{\frac{1}{2}}}^{+\infty} \int_{0}^{+\infty} t^{N-1} G\left(\frac{1}{t^{N-2}} \frac{A}{\left(1+r^{2}\right)^{\frac{N-2}{2}}}\right) r^{N-2} d r d t .
$$

Then (2.45) and (2.46) are a consequence of (2.41) and (2.49).

When $H\left(x_{0}\right)>0$.

\section{Lemma 2.3}

Assume that $f(x, u)$ satisfies (2.1)-(2.5) and (2.7) or (2.1)-(2.4) and (2.6)-(2.7). Suppose that there exists some continuous function $g$ such that

$$
g(x, u) \geq g(u) \text { for a.e. } x \in \Omega \text { and for all } u \in \mathbb{R}
$$


and the primitive $G(u)=\int_{0}^{u} g(t) d t$ satisfies, for $N \geq 4$

$$
\lim _{\varepsilon \rightarrow 0} \varepsilon^{\frac{N-2}{2}} \int_{\varepsilon^{\frac{1}{2}}}^{+\infty} t^{N-1} \int_{0}^{+\infty} G\left(\frac{t^{-(N-2)}}{\left(1+r^{2}\right)^{\frac{(N-2)}{2}}}\right) r^{N-2} d r d t=0 .
$$

and for $N=3$

$$
\lim _{\varepsilon \rightarrow 0} \frac{\varepsilon^{\frac{1}{2}}}{|\ln (\varepsilon)|} \int_{\varepsilon^{\frac{1}{2}}}^{+\infty} t^{2} \int_{0}^{+\infty} G\left(\frac{t^{-1}}{\left(1+r^{2}\right)^{\frac{1}{2}}}\right) r d r d t=0 .
$$

Then condition (2.8) holds.

\section{Proof.}

The proof of this Lemma is similar to proof of Lemma 2.2 .

Now let us give some examples for the nonlinear perturbation.

\section{Examples of $f$ :}

If $H\left(x_{0}\right)>0$ then the two functions $g$ below satisfy the hypothesis of Lemma 2.2 .

1) $g(x, u)=g(u)=\mu|u|^{r-2} u$ with $\mu>0$ and $2_{*}<r<2^{*}$.

2)

$$
g(x, u)=g(u)=\left\{\begin{array}{l}
(3+\gamma) u^{2+\gamma} \ln (u)+|u|^{2+\gamma} \quad \text { if } u>1 \\
(3+\gamma)|u|^{2+\gamma}|\ln (u)|+|u|^{2+\gamma} \quad \text { if } u<1
\end{array}\right.
$$

with $0<\gamma<\frac{2}{N-2}$

If $H\left(x_{0}\right)<0$ then the two functions $g$ below satisfy Lemma 2.3.

1) $g(x, u)=g(u)=\mu|u|^{r-2} u$ with $\mu \in \mathbb{R}$ and $2<r<2$.

2) $g(x, u)=g(u)= \pm \frac{5}{2} \frac{|u|^{\frac{3}{2}}+|u|^{\frac{7}{2}}}{\left(1+5|u|^{2}\right)^{2}}$.

\section{Existence results in presence of two critical exponents.}

We assume that $\beta=1$ and, as in the previous section, the nonlinearity $f(x, u)$ satisfies the following basic assumptions.

$$
f(x, u)=a(x) u+g(x, u)
$$

with

$$
\begin{gathered}
a(x) \in L^{\infty}(\Omega), \\
|g(x, u)|=o(|u|) \quad \text { as } \quad u \rightarrow 0, \quad \text { uniformly in } x, \\
|g(x, u)|=o\left(|u|^{2^{*}-1}\right) \quad \text { as }|u| \rightarrow+\infty, \quad \text { uniformly in } x .
\end{gathered}
$$


Moreover we assume that

$$
\lambda_{1}(a)=\inf \left\{\int_{\Omega}|\nabla u|^{2}-a(x) u^{2} d x, \int_{\Omega} u^{2} d x=1\right\}>0 .
$$

Set $F(x, u)=\int_{0}^{u} f(x, t) d t$ for $x \in \bar{\Omega}, u \in \mathbb{R}$. Let define, for $u \in H^{1}(\Omega)$,

$$
\Phi(u)=\frac{1}{2} \int_{\Omega} p(x)|\nabla u|^{2} d x-\frac{1}{2^{*}} \int_{\Omega}|u|^{2^{*}} d x-\frac{1}{2_{*}} \int_{\partial \Omega} p(x) Q(x)|u|^{2_{*}} d s_{x}-\int_{\Omega} F(x, u) d x
$$

Our main result in this section is

\section{Theorem 3.1}

Assume (3.1)-(3.5) and suppose, moreover, that

$$
\begin{aligned}
& \text { there exists some } v_{0} \in H^{1}, v_{0} \geq 0 \text { on } \Omega, v_{0} \neq 0 \text { on } \partial \Omega \text {, such that } \\
& \sup _{t \geq 0} \Phi\left(t v_{0}\right)<M\left(S, S_{1}\right), \quad \text { where } M\left(S, S_{1}\right) \text { is defined in (1.4). }
\end{aligned}
$$

Then, problem (1.1) possesses a solution.

\section{Proof of Theorem 3.1.}

From (3.3) we have, for any $\varepsilon>0$, there is a $\delta>0$ such that

$$
|g(x, u)| \leq \varepsilon|u| \text { for a.e } x \in \Omega \text {, and for all }|u| \leq \delta \text {, }
$$

thus, by (3.4), we obtain

$$
|g(x, u)| \leq \varepsilon|u|+C|u|^{2^{*}-1} \quad \text { for a.e } x \in \Omega, \text { and for all } u \in \mathbb{R},
$$

and for some constant $C$ (depending on $\varepsilon$ ). Therefore we have

$$
G(x, u) \leq \frac{1}{2} a(x) u^{2}+\frac{\varepsilon}{2} u^{2}+\frac{C}{2^{*}}|u|^{2^{*}} \quad \text { for a.e } x \in \Omega, \text { and for all } u \in \mathbb{R} .
$$

Therefore, for all $u \in H^{1}(\Omega)$,

$\Phi(u) \geq \frac{1}{2} \int_{\Omega} p(x)|\nabla u|^{2} d x-\frac{1}{2} \int_{\Omega} a(x)|u|^{2} d x-\frac{\varepsilon}{2} \int_{\Omega}|u|^{2} d x-\frac{C}{2^{*}} \int_{\Omega}|u|^{2^{*}} d x-\frac{1}{2_{*}} \int_{\partial \Omega} p(x) Q(x)|u|^{2 *} d s_{x}$

Using (3.5) we easily see that, for $\varepsilon>0$ small enough, there exist constants $k>0$, $C_{1}>0$ and $C_{2}>0$ such that

$$
\begin{aligned}
\Phi(u) & \geq k\|u\|_{H^{1}}^{2}-C_{1}\|u\|_{H^{1}}^{2^{*}}-C_{2}\|u\|_{H^{1}}^{2 *} \\
& \geq\|u\|_{H^{1}}^{2}\left(k-C_{1}\|u\|_{H^{1}}^{2^{*}-2}-C_{2}\|u\|_{H^{1}}^{2_{*}-2}\right) \quad \text { for all } u \in H^{1} .
\end{aligned}
$$

Which implies, since $2_{*}>2$ and $2^{*}>2$, that for some small $\alpha>0$ there exists $\rho>0$ such that

$$
\Phi(u) \geq \rho, \quad \text { provided }\|u\|=\alpha .
$$

On the other hand, for any $u \in H^{1}(\Omega), u \neq \equiv 0$ in $\bar{\Omega}$, we have by (3.4) $\lim _{t \rightarrow+\infty} \Phi(t u)=$ $-\infty$. Thus for later purpose we take $v=t_{0} U_{\varepsilon, x_{0}}$, where $t_{0}>0$ is chosen large enough so 
that $v \notin U$ and $\Phi(v) \leq 0$.

Set

$$
c=\inf _{\mathcal{P} \in \mathcal{A}} \max _{w \in \mathcal{P}} \Phi(w),
$$

where $\mathcal{A}$ denotes the class of continuous paths joining 0 to $v$.

Looking at (3.7) we see that $c<M\left(S, S_{1}\right)$.

By a result of Ambrosetti and Rabinowtz [2], see also [4], there exists a sequence $\left\{u_{j}\right\}$ in $H^{1}(\Omega)$ satisfying

$$
\Phi\left(u_{j}\right) \rightarrow c<M\left(S, S_{1}\right)
$$

and

$$
\Phi^{\prime}\left(u_{j}\right) \rightarrow 0 \quad \text { in } H^{-1}(\Omega)
$$

Using (3.1) and (3.5), from (3.10) and (3.11) we write

$$
\frac{1}{2}\left\|u_{j}\right\|^{2}-\frac{1}{2^{*}} \int_{\Omega}\left|u_{j}\right|^{2^{*}} d x-\frac{1}{2_{*}} \int_{\partial \Omega} p(x) Q(x)\left|u_{j}\right|^{2 *} d s_{x}-\int_{\Omega} G\left(x, u_{j}\right)=c+o(1),
$$

and

$$
\left\|u_{j}\right\|^{2}-\int_{\Omega}\left|u_{j}\right|^{2^{*}} d x-\int_{\partial \Omega} p(x) Q(x)\left|u_{j}\right|^{2_{*}} d s_{x}-\int_{\Omega} g\left(x, u_{j}\right) u_{j} d x=<\xi_{j}, u_{j}>
$$

with $\xi_{j} \rightarrow 0$ in $H^{-1}$.

We start by showing that $\left\{u_{j}\right\}$ is bounded in $H^{1}(\Omega)$.

Computing (3.12) $-\frac{1}{2_{*}}(3.13)$, we obtain

$\frac{1}{2(N-1)}\left\|u_{j}\right\|^{2}+\frac{N-2}{2 N(N-1)} \int_{\Omega}\left|u_{j}\right|^{2^{*}} d x-\int_{\Omega}\left[G\left(x, u_{j}\right)-\frac{1}{2_{*}} g\left(x, u_{j}\right) u_{j}\right] d x=c+o(1)+<\xi_{j}, u_{j}>$.

On the other hand, from (3.4) we have for all $\varepsilon>0$ there exists $C>0$ such that

$$
|g(x, u)| \leq \varepsilon|u|^{2^{*}-1}+C \quad \text { for a.e } x \in \Omega \text { and for all } u \in \mathbb{R},
$$

and therefore

$$
|G(x, u)| \leq \frac{\varepsilon}{2^{*}}|u|^{2^{*}}+C u \quad \text { for a.e } x \in \Omega \text { and for all } u \in \mathbb{R} .
$$

We deduce from (3.14)-(3.16), after using the embedding $L^{2}(\Omega) \hookrightarrow L^{1}(\Omega)$ and $H^{1}(\Omega) \hookrightarrow$ $L^{2}(\Omega)$ that, for $\varepsilon>0$ small enough,

$$
\frac{1}{2(N-1)}\left\|u_{j}\right\|^{2}+\frac{N-2}{2 N(N-1)}(1+\varepsilon) \int_{\Omega}\left|u_{j}\right|^{2^{*}} d x-C^{\prime}\left\|u_{j}\right\| \leq c+o(1)
$$

for some constant $C^{\prime}>0$. This gives that $\left\{u_{j}\right\}$ is bounded in $H^{1}(\Omega)$, otherwise we obtain a contradiction. 
Extract a subsequence, still denoted by $u_{j}$, such that

$$
\begin{aligned}
u_{j} & \rightarrow u \quad \text { weakly in } H^{1}(\Omega), \\
u_{j} & \rightarrow u \quad \text { strongly in } L^{t}(\Omega) \text { for all } t<2^{*}=\frac{2 N}{N-2}, \\
u_{j} & \rightarrow u \quad \text { a.e. on } \Omega, \\
f\left(x, u_{j}\right) & \rightarrow f(x, u) \quad \text { weakly in } L^{\frac{2^{*}}{2^{*}-1}}(\Omega), \\
u_{j} & \rightarrow u \quad \text { weakly in } L^{2^{*}}(\partial \Omega), \\
u_{j} & \rightarrow u \quad \text { weakly in } L^{2^{*}}(\Omega) .
\end{aligned}
$$

We shall now verify that $u \not \equiv 0$ on $\Omega$.

Indeed, suppose that $u \equiv 0$. We claim that

$$
\int_{\Omega} f\left(x, u_{j}\right) u_{j} d x \rightarrow 0 \text { and } \int_{\Omega} F\left(x, u_{j}\right) d x \rightarrow 0 .
$$

From (3.15) and (3.16), we have, for all $\varepsilon>0$ there exists $C>0$ such that

$$
\left|\int_{\Omega} f\left(x, u_{j}\right) u_{j} d x\right| \leq \varepsilon \int_{\Omega}\left|u_{j}\right|^{2^{*}} d x+C \int_{\Omega}\left|u_{j}\right| d x
$$

and

$$
\left|\int_{\Omega} F\left(x, u_{j}^{+}\right) d x\right| \leq \frac{\varepsilon}{2^{*}} \int_{\Omega}\left|u_{j}\right|^{2^{*}} d x+\frac{C}{2} \int_{\Omega}\left|u_{j}\right|^{2} d x .
$$

Since $\left\{u_{j}\right\}$ remains bounded in $L^{2^{*}}(\Omega)$ and $u_{j} \rightarrow 0$ in $L^{2}(\Omega)$ we obtain (3.17).

Now, extruding a subsequence, still denoted by $u_{j}$, we may assume that there exist some constants $l \geq 0, m_{1} \geq 0$ and $m_{2} \geq 0$ such that

$$
\int_{\Omega} p(x)\left|\nabla u_{j}\right|^{2} d x \rightarrow l, \quad \int_{\Omega}\left|u_{j}\right|^{2^{*}} d x \rightarrow m_{1}, \quad \text { and } \quad \int_{\partial \Omega} p(x) Q(x)\left|u_{j}\right|^{2 *} d s_{x} \rightarrow m_{2} .
$$

Passing to the limit in (3.12) and (3.13), we get

$$
\frac{1}{2} l-\frac{1}{2^{*}} m_{1}-\frac{1}{2_{*}} m_{2}=c \quad \text { and } \quad l-m_{1}-m_{2}=0 .
$$

From the result of $[24$, Theorem 01], we know that there exists a constant $C(\Omega)>0$ such that for every $w \in H^{1}(\Omega)$

$$
\int_{\Omega}|\nabla w|^{2} d x+C(\Omega) \int_{\Omega}|w|^{k} d x \geq \frac{S}{2^{\frac{2}{N}}}\left(\int_{\Omega}|w|^{2 *} d x\right)^{\frac{2}{2^{*}}}
$$

with $k=\frac{2 N}{N-1}$ if $N \geq 4$ and $k>3=\frac{2 N}{N-1}$ if $N=3$.

We apply this result for $w_{j}=(p(x))^{\frac{1}{2}} u_{j}$ and in particular for $N=3$ we take $k$ such that $6=\frac{2 N}{N-2}>k>3$, we obtain for $j$ large enough

$$
\int_{\Omega}\left|\nabla(p(x))^{\frac{1}{2}} u_{j}\right|^{2} d x+C(\Omega) \int_{\Omega}\left|(p(x))^{\frac{1}{2}} u_{j}\right|^{k} d x \geq \frac{S}{2^{\frac{2}{N}}}\left(\int_{\Omega}\left|(p(x))^{\frac{1}{2}} u_{j}\right|^{2^{*}} d x\right)^{\frac{2}{2^{*}}}
$$


Since $k<\frac{2 N}{N-2}$ for every $N \geq 3$, thanks to the compact embedding $H^{1}(\Omega) \hookrightarrow L^{k}(\Omega)$, we have $u_{j} \rightarrow 0$ strongly in $L^{k}(\Omega)$ and we deduce

$$
\int_{\Omega} p(x)\left|\nabla u_{j}\right|^{2} d x+o(1) \geq \frac{S}{2^{\frac{2}{N}}}\left(\int_{\Omega}\left|(p(x))^{\frac{1}{2}} u_{j}\right|^{2_{*}} d x\right)^{\frac{2}{2^{*}}}+o(1) .
$$

Using the fact that $p(x) \geq p_{0}$ for all $x \in \bar{\Omega}$, we see that

$$
\int_{\Omega} p(x)\left|\nabla u_{j}\right|^{2} d x+o(1) \geq \frac{p_{0} S}{2^{\frac{2}{N}}}\left(\int_{\Omega}\left|u_{j}\right|^{2_{*}} d x\right)^{\frac{2}{2^{*}}}+o(1) .
$$

At the limit we obtain

$$
\left(m_{1}\right)^{\frac{2}{2^{*}}} \frac{p_{0} S}{2^{\frac{2}{N}}} \leq l
$$

On the other hand, by the same way, from [24, Theorem 02] we have (see (2.29) for more details)

$$
\left(m_{2}\right)^{\frac{2}{2_{*}}}\left[\frac{p\left(x_{0}\right)}{\left(Q\left(x_{0}\right)\right)^{N-2}}\right]^{\frac{1}{N-1}} S_{1} \leq l .
$$

Combining (3.19), (3.20) and (3.21) we obtain the following

$$
\left\{\begin{array}{l}
\frac{1}{2(N-1)} l+\frac{N-2}{2 N(N-1)} m_{1}=c \\
\frac{1}{N} l-\frac{N-2}{2 N(N-1)} m_{2}=c \\
m_{1} \leq\left(\frac{2^{\frac{2}{N}} l}{p(a) S}\right)^{\frac{2^{*}}{2}} \\
m_{2} \leq\left(\frac{l}{\left[\frac{p\left(x_{0}\right)}{\left(Q\left(x_{0}\right)\right)^{N-2}}\right]^{\frac{1}{N-1}} S_{1}}\right)^{\frac{2_{*}}{2}} .
\end{array}\right.
$$

An easy computation yields

$$
\frac{1}{N} l-\frac{N-2}{2(N-1) N}\left(\frac{l}{\frac{p\left(x_{0}\right)}{\left(Q\left(x_{0}\right)\right)^{N-2}} S_{1}}\right)^{\frac{2 *}{2}} \leq c \leq \frac{1}{2(N-1)} l+\frac{N-2}{2(N-1) N}\left(\frac{2^{\frac{2}{N}} l}{p_{0} S}\right)^{\frac{2^{*}}{2}} .
$$

We can write

$$
l \leq\left(\frac{l}{\frac{p\left(x_{0}\right)}{\left(Q\left(x_{0}\right)\right)^{N-2}} S_{1}}\right)^{\frac{2_{*}}{2}}+\left(\frac{2^{\frac{2}{N}} l}{p_{0} S}\right)^{\frac{2^{*}}{2}}
$$

If $l=0$ then, since $c>0$, we obtain a contradiction and we get the desired result. Now, if $l \neq 0$ we reduce to the study of the following polynomial

$$
\frac{1}{\left(2^{-\frac{2}{N}} p_{0} S\right)^{\frac{N}{N-2}}} t^{2}+\frac{1}{\left(\frac{p\left(x_{0}\right)}{\left(Q\left(x_{0}\right)\right)^{N-2}} S_{1}\right)^{\frac{N-1}{N-2}}} t-1 \geq 0 \quad \text { where } t=l^{\frac{1}{N-2}} .
$$

Which is possible if $t \geq \frac{2\left(\frac{p\left(x_{0}\right)}{\left(Q\left(x_{0}\right)\right)^{N-2}} S_{1}\right)^{\frac{N-1}{N-2}}}{1+\sqrt{1+4 E^{\prime}}}$ where $E^{\prime}=\left(\frac{\frac{p\left(x_{0}\right)}{\left(Q\left(x_{0}\right)\right)^{N-2}} S_{1}^{N-1}}{\left(2^{-\frac{2}{N}} p_{0} S\right)^{\frac{N}{2}}}\right)^{\frac{2}{N-2}}$.

From the left inequality of (3.23) and the fact that $l=t^{N-2}$, we obtain $c \geq M\left(S, S_{1}\right)$ which gives a contradiction with (3.10). Consequently $u \not \equiv 0$ and $u$ is a solution of (1.1). 


\section{Remark 3.1}

If we assume that

$$
F(x, v) \leq \frac{1}{2} f(x, v) v+\frac{1}{N}|v|^{2^{*}}, \quad \text { for all } v \in \mathbb{R} \text { and for for a.e } x \in \Omega .
$$

then the previous sequence $\left\{u_{j}\right\}$ is relatively compact in $H^{1}(\Omega)$.

Let $\left\{u_{j}\right\}$ be the sequence defined in the proof of Theorem 3.1, we recall that $u_{j}$ converge weakly to $u$ in $H^{1}(\Omega)$. We will show that $u_{j}$ converges strongly to $u$ in $H^{1}(\Omega)$. Firstly, since $u$ is a solution of (1.1), we have

$$
\int_{\Omega} p(x)|\nabla u|^{2} d x=\int_{\Omega}|u|^{2^{*}} d x+\int_{\Omega} f(x, u) u d x+\int_{\partial \Omega} p(x) Q(x)|u|^{2_{*}} d s_{x} .
$$

Therefore

$$
\Phi(u)=\int_{\Omega}\left\{\frac{1}{N}|u|^{2^{*}}+\frac{1}{2} f(x, u) u-F(x, u)\right\} d x+\frac{1}{2(N-1)} \int_{\partial \Omega} p(x) Q(x)|u|^{2 *} d s_{x} .
$$

Using (3.24) we have $\Phi(u) \geq 0$.

Now, we set $v_{j}=u_{j}-u$.

We write

$$
\int_{\Omega} p(x)\left|\nabla u_{j}\right|^{2} d x=\int_{\Omega} p(x)|\nabla u|^{2} d x+\int_{\Omega} p(x)\left|\nabla v_{j}\right|^{2} d x+o(1)
$$

and from [5] we deduce that

$$
\int_{\Omega}\left|u_{j}\right|^{2^{*}} d x=\int_{\Omega}|u|^{2^{*}} d x+\int_{\Omega}\left|v_{j}\right|^{2^{*}} d x+o(1)
$$

and

$$
\int_{\partial \Omega} p(x) Q(x)\left|u_{j}\right|^{2_{*}} d s_{x}=\int_{\partial \Omega} p(x) Q(x)|u|^{2 *} d s_{x}+\int_{\partial \Omega} p(x) Q(x)\left|v_{j}\right|^{2 *} d s_{x}+o(1)
$$

Inserting (3.25), (3.26) and (3.27) into (3.12) and (3.13) we get

$$
\Phi(u)+\frac{1}{2} \int_{\Omega} p(x)\left|\nabla v_{j}\right|^{2} d x-\frac{1}{2^{*}} \int_{\Omega}\left|v_{j}\right|^{2^{*}} d x-\frac{1}{2_{*}} \int_{\partial \Omega} p(x) Q(x)\left|v_{j}\right|^{2 *} d s_{x}=c+o(1)
$$

and (looking at (3.11))

$$
\int_{\Omega} p(x)\left|\nabla v_{j}\right|^{2} d x-\int_{\Omega}\left|v_{j}\right|^{2^{*}} d x-\int_{\partial \Omega} p(x) Q(x)\left|v_{j}\right|^{2 *} d s_{x}=o(1) .
$$

Now, we assume (for a subsequence) that exists some constants $l \geq 0, m_{1} \geq 0$ and $m_{2} \geq 0$ such that

$$
\int_{\Omega} p(x)\left|\nabla v_{j}\right|^{2} d x \rightarrow l, \quad \int_{\Omega}\left|v_{j}\right|^{2^{*}} d x \rightarrow m_{1} \quad \text { and } \quad \int_{\partial \Omega} p(x) Q(x)\left|v_{j}\right|^{2 *} d s_{x} \rightarrow m_{2} .
$$


Passing to limit in (3.28) and (3.29), using the Sobolev embedding, a easy computation yields

$$
\left\{\begin{array}{l}
\frac{1}{2(N-1)} l+\frac{N-2}{2 N(N-1)} m_{1}=c-\Phi(u) \\
\frac{1}{N} l-\frac{N-2}{2 N(N-1)} m_{2}=c-\Phi(u) \\
m_{1} \leq\left(\frac{l}{p(a) S}\right)^{\frac{2^{*}}{2}} \\
m_{2} \leq\left(\frac{l}{\left[\frac{p\left(x_{0}\right)}{\left(Q\left(x_{0}\right)\right)^{N-2}}\right]^{\frac{1}{N-1}} S_{1}}\right)^{\frac{2_{*}}{2}} .
\end{array}\right.
$$

Therefore, as in end of proof of Theorem 3.1, if $l \neq 0$ then $c-\Phi(u) \geq M\left(S, S_{1}\right)$ which is a contradiction since $c<M\left(S, S_{1}\right)$ and $\Phi(u) \geq 0$. Consequently $l=0$ and then $u_{j} \rightarrow u$ strongly in $H^{1}(\Omega)$.

\subsection{Sufficient conditions on $f(x, u)$ which give condition (3.7):}

We recall

$$
S=\inf \left\{\int_{\mathbb{R}^{N}}|\nabla u|^{2} d x ; u \in H^{1}\left(\mathbb{R}^{N}\right), \int_{\mathbb{R}^{N}}|u|^{2^{*}} d x=1\right\} .
$$

We consider, for all $\varepsilon>0$, the following functions

$$
U_{\varepsilon, y}(x)=\left(\frac{\varepsilon}{\varepsilon^{2}+\left|x^{\prime}-y^{\prime}\right|^{2}+\left|x_{N}-y_{N}+\mu(N-2)^{-1} \varepsilon\right|^{2}}\right)^{\frac{N-2}{2}},
$$

where $\left.x=\left(x^{\prime}, x_{N}\right), y=\left(y^{\prime}, y_{N}\right) \in \mathbb{R}^{N-1} \times\right] 0,+\infty\left[, \mu \in \mathbb{R}\right.$ and $u_{\varepsilon, x_{0}}=\xi(x) U_{\varepsilon, x_{0}}(x)$, where $\xi$ be a radial $C^{\infty}$-function such that, for a fixed positive constant $R$,

$$
\xi(x)=\left\{\begin{array}{l}
1 \text { if }\left|x-x_{0}\right| \leq \frac{R}{4} \\
0 \text { if }\left|x-x_{0}\right|>\frac{R}{2}
\end{array}\right.
$$

It is known, see [12] and [23], that $U_{\varepsilon, y}$ is a solution of the following problem

$$
\begin{cases}-\Delta u=N(N-2) u^{\frac{N+2}{N-2}} & \text { in } \mathbb{R}_{+}^{n} \\ u>0 & \text { in } \mathbb{R}_{+}^{N} \\ -\frac{\partial u}{\partial x_{N}}=\mu u^{\frac{N}{N-2}} & \text { on } \partial \mathbb{R}_{+}^{N}=\mathbb{R}^{N-1} .\end{cases}
$$

We draw on estimates made in [11, pages 17-22], we write

$$
\int_{\Omega} p(x)\left|\nabla u_{\varepsilon, x_{0}}\right|^{2} d x=p\left(x_{0}\right) A_{\mu}-\mu H\left(x_{0}\right) p\left(x_{0}\right) \begin{cases}K_{1} \varepsilon+o(\varepsilon) & \text { if } N \geq 4 \\ K_{0} \varepsilon|\log \varepsilon|+o(\varepsilon|\log \varepsilon|) & \text { If } N=3,\end{cases}
$$

$$
\int_{\Omega}\left|u_{\varepsilon, x_{0}}\right|^{2^{*}} d x=B_{\mu}-\mu H\left(x_{0}\right) K_{2} \varepsilon+o(\varepsilon) \quad \text { for all } N \geq 3
$$

$\int_{\partial \Omega} p(x) Q(x)\left|u_{\varepsilon, x_{0}}\right|^{2 *} d s_{x}=p\left(x_{0}\right) Q\left(x_{0}\right) C_{\mu}+\mu H\left(x_{0}\right) p\left(x_{0}\right) Q\left(x_{0}\right) K_{3} \varepsilon+o(\varepsilon) \quad$ for all $N \geq 3$, 
where $A_{\mu}, B_{\mu}, C_{\mu}$ and $K_{i}>0$ for $i \in\{0,1,2,3\}$ are defined by

$$
\begin{gathered}
A_{\mu}=\int_{\mathbb{R}_{+}^{N}}\left|\nabla U_{\varepsilon, x_{0}}\right|^{2} d x=\int_{\frac{\mu}{N-2}}^{+\infty} \int_{\mathbb{R}^{N-1}} \frac{|x|^{2}}{\left(1+|x|^{2}\right)^{N}} d x \\
B_{\mu}=\int_{\mathbb{R}_{+}^{N}}\left|U_{\varepsilon, x_{0}}\right|^{2^{*}} d x=\int_{\frac{\mu}{N-2}}^{+\infty} \int_{\mathbb{R}^{N-1}} \frac{1}{\left(1+|x|^{2}\right)^{N}} d x \\
C_{\mu}=\int_{R^{N-1}}\left|U_{\varepsilon, x_{0}}\right|^{2 *} d x^{\prime}=\frac{1}{\left(1+\left(\frac{\mu}{N-2}\right)^{2}\right)^{\frac{N-2}{2}}} \int_{\mathbb{R}^{N-1}} \frac{1}{\left(1+|y|^{2}\right)^{N-1}} d y, \\
K_{1}=(N-2)^{2}\left(\frac{N+1}{N-3}+2 \frac{N-1}{N-3} \mu^{2}\right) K_{2}, \\
K_{3}=2(N-1) \mu K_{2}
\end{gathered}
$$

with $K_{2}>0$ and $K_{0}>0$ are some constants. Let

$$
J(u)=\frac{1}{2} p\left(x_{0}\right) \int_{\mathbb{R}_{+}^{N}}|\nabla u|^{2} d x-\frac{1}{2^{*}} \int_{\mathbb{R}_{+}^{N}}|u|^{2^{*}} d x-\frac{1}{2_{*}} p\left(x_{0}\right) Q\left(x_{0}\right) \int_{R^{N-1}}\left|u\left(x^{\prime}, 0\right)\right|^{2 *} d x^{\prime},
$$

We have the following result

Proposition 3.2 We have

$$
\inf _{u \in H^{1}\left(\mathbb{R}_{+}^{N}\right) \backslash\{0\}} \max _{t \geq 0} J(t u) \leq M\left(S, S_{1}\right), \quad \text { where } M\left(S, S_{1}\right) \text { is defined in (1.4). }
$$

\section{Proof.}

We have

$$
J\left(t U_{\varepsilon, 0}\right)=\frac{t^{2}}{2} p\left(x_{0}\right) A_{\mu}-\frac{t^{2^{*}}}{2^{*}} B_{\mu}-\frac{t^{2 *}}{2_{*}} p\left(x_{0}\right) Q\left(x_{0}\right) C_{\mu} .
$$

Set $h(t)=\frac{t^{2}}{2} p\left(x_{0}\right) A_{\mu}-\frac{t^{2^{*}}}{2^{*}} B_{\mu}-\frac{t^{2 *}}{2_{*}} p\left(x_{0}\right) Q\left(x_{0}\right) C_{\mu}$.

Therefore

$$
\max _{t \geq 0} J\left(t U_{\varepsilon, 0}\right)=\max _{t \geq 0} h(t) .
$$

Let $t_{\mu}$ such that $h\left(t_{\mu}\right)=\max _{t \geq 0} h(t)$. Then $t_{\mu}$ satisfies

$$
p\left(x_{0}\right) A_{\mu}-B_{\mu} t_{\mu}^{\frac{4}{N-2}}-p\left(x_{0}\right) Q\left(x_{0}\right) C_{\mu} t_{\mu}^{\frac{2}{N-2}}=0 .
$$

Looking at the polynomial $B_{\mu} l^{2}+p\left(x_{0}\right) Q\left(x_{0}\right) C_{\mu} l-p\left(x_{0}\right) A_{\mu}$ we deduce that

$$
\begin{aligned}
t_{\mu} & =\left[\frac{-p\left(x_{0}\right) Q\left(x_{0}\right) C_{\mu}+\sqrt{\left(p\left(x_{0}\right) Q\left(x_{0}\right) C_{\mu}\right)^{2}+4 p\left(x_{0}\right) A_{\mu} B_{\mu}}}{2 B_{\mu}}\right]^{\frac{N-2}{2}} \\
& =2^{\frac{N-2}{2}}\left(\frac{A_{\mu}}{Q\left(x_{0}\right) C_{\mu}}\right)^{\frac{N-2}{2}} \frac{1}{\left[1+\sqrt{1+4 \frac{p\left(x_{0}\right) A_{\mu} B_{\mu}}{\left(Q\left(x_{0}\right) C_{\mu}\right)^{2}}}\right]^{\frac{N-2}{2}}} .
\end{aligned}
$$


Hence

$$
h\left(t_{\mu}\right)=t_{\mu}^{2}\left[\frac{p\left(x_{0}\right) A_{\mu}}{N}-\frac{N-2}{2 N(N-1)} p\left(x_{0}\right) Q\left(x_{0}\right) C_{\mu} t_{\mu}^{\frac{2}{N-2}}\right] .
$$

By a standard computation we have

$$
h\left(t_{\mu}\right)=A_{\mu}\left(\frac{\left(\frac{2 A_{0}}{Q\left(x_{0}\right) C_{0}}\right)}{1+\sqrt{1+4 \frac{A_{\mu} B_{\mu}}{p\left(x_{0}\right)\left(Q\left(x_{0}\right)\right)^{2} C_{\mu}^{2}}}}\right)^{N-2}\left[\frac{1}{N}-\frac{N-2}{N(N-1)} \frac{1}{1+\sqrt{1+4 \frac{A_{\mu} B_{\mu}}{p\left(x_{0}\right)\left(Q\left(x_{0}\right)\right)^{2} C_{\mu}^{2}}}}\right]
$$

From (3.35)-(3.37) we see, for $\mu>0$ small enough, that

$$
\begin{aligned}
h\left(t_{\mu}\right) & =A_{0}\left(\frac{\left(\frac{2 A_{0}}{Q\left(x_{0}\right) C_{0}}\right)}{1+\sqrt{1+4 \frac{A_{0} B_{0}}{p\left(x_{0}\right)\left(Q\left(x_{0}\right)\right)^{2} C_{0}^{2}}}}\right)^{N-2}\left[\frac{1}{N}-\frac{N-2}{N(N-1)} \frac{1}{1+\sqrt{1+4 \frac{A_{0} B_{0}}{p\left(x_{0}\right)\left(Q\left(x_{0}\right)\right)^{2} C_{0}^{2}}}}\right] \\
& +\mu L+o(\mu),
\end{aligned}
$$

where $L$ is a constant.

Using the fact that $S_{1}=\frac{A_{0}}{\left(C_{0}\right)^{\frac{2}{2 *}}}, S=\frac{A_{\infty}}{\left(B_{\infty}\right)^{\frac{2}{2^{*}}}}, A_{\infty}=2 A_{0}$ and $B_{\infty}=2 B_{0}$, we obtain, for $\mu>0$ small enough, that

$$
\max _{t \geq 0} J\left(t U_{\varepsilon, 0}\right)=h\left(t_{\mu}\right)=M\left(S, S_{1}\right)+\mu L+o(\mu) .
$$

This gives the desired result.

Now, we will show, under some additional conditions on $f(x, u)$, that $u_{\varepsilon, x_{0}}$, defined by (3.30), satisfies condition (3.7).

We have

$$
\begin{aligned}
\Phi\left(t u_{\varepsilon, x_{0}}\right) & =\frac{1}{2} t^{2} \int_{\Omega} p(x)\left|\nabla u_{\varepsilon, x_{0}}\right|^{2} d x-\frac{t^{2^{*}}}{2^{*}} \int_{\Omega}\left|u_{\varepsilon, x_{0}}\right|^{2^{*}} d x-\frac{t^{2 *}}{2_{*}} \int_{\partial \Omega} p(x) Q(x)\left|u_{\varepsilon, x_{0}}\right|^{2 *} d s_{x} \\
& -\int_{\Omega} F\left(x, t u_{\varepsilon, x_{0}}\right) d x .
\end{aligned}
$$

Since $f(x, u)$ is a lower-order perturbation of $|u|^{2^{*}-1}$, we see that $\lim _{t \rightarrow+\infty} \Phi\left(t u_{\varepsilon, x_{0}}\right)=-\infty$. Therefore $\sup _{t \geq 0} \Phi\left(t u_{\varepsilon, x_{0}}\right)$ is achieved at some $t_{\varepsilon} \geq 0$ and $t_{\varepsilon}$ is bounded in $\mathbb{R}_{+}$.

From now we suppose that $t_{\varepsilon}>0$, otherwise condition (3.7) is easily satisfied.

We write $t_{\varepsilon}=t_{0}+O(\varepsilon)$ when $N \geq 4$ and $t_{\varepsilon}=t_{0}+O(\varepsilon|\ln (\varepsilon)|)$ when $N=3$, using (3.32)-(3.34) we get

$$
\text { If } N \geq 4 \text { : }
$$

$$
\begin{aligned}
\Phi\left(t_{\varepsilon} u_{\varepsilon, x_{0}}\right) & =\frac{t_{\varepsilon}^{2}}{2} p\left(x_{0}\right) \int_{\mathbb{R}_{+}^{N}}\left|\nabla U_{\varepsilon, 0}\right|^{2} d x-\frac{t_{\varepsilon}^{2^{*}}}{2} \int_{\mathbb{R}_{+}^{N}}\left|U_{\varepsilon, 0}\right|^{2^{*}} d x \\
& -\frac{t_{\varepsilon}^{2_{*}}}{2} p\left(x_{0}\right) Q\left(x_{0}\right) \int_{R^{N-1}}\left|U_{\varepsilon, 0}\left(x^{\prime}, 0\right)\right|^{2_{*}} d x^{\prime}-\frac{t_{0}^{2}}{2} \mu H\left(x_{0}\right) K_{1} \varepsilon+\frac{t_{0}^{2^{*}}}{2^{*}} \mu H\left(x_{0}\right) K_{2} \varepsilon \\
& -\frac{t_{0}^{2_{*}}}{2_{*}} \mu H\left(x_{0}\right) K_{3} \varepsilon-\int_{\Omega} F\left(x, t u_{\varepsilon, x_{0}}\right) d x+o(\varepsilon) \\
& \leq \max _{t \geq 0} J\left(t U_{\varepsilon, 0}\right)-\frac{t_{0}^{2}}{2} \mu H\left(x_{0}\right) p\left(x_{0}\right) K_{1} \varepsilon+\frac{t_{0}^{2^{*}}}{2^{*}} \mu H\left(x_{0}\right) K_{2} \varepsilon \\
& -\frac{t_{0}^{2 *}}{2_{*}} \mu H\left(x_{0}\right) p\left(x_{0}\right) Q\left(x_{0}\right) K_{3} \varepsilon-\int_{\Omega} F\left(x, t u_{\varepsilon, x_{0}}\right) d x+o(\varepsilon)
\end{aligned}
$$


If $N=3$ :

$$
\begin{aligned}
\Phi\left(t_{\varepsilon} u_{\varepsilon, x_{0}}\right) & =\frac{t_{\varepsilon}^{2}}{2} p\left(x_{0}\right) \int_{\mathbb{R}_{+}^{N}}\left|\nabla U_{\varepsilon, 0}\right|^{2} d x-\frac{t_{\varepsilon}^{2^{*}}}{2} \int_{\mathbb{R}_{+}^{N}}\left|U_{\varepsilon, 0}\right|^{2^{*}} d x \\
& -\frac{t_{\varepsilon}^{2 *}}{2} p\left(x_{0}\right) Q\left(x_{0}\right) \int_{R^{N-1}}\left|U_{\varepsilon, 0}\left(x^{\prime}, 0\right)\right|^{2_{*}} d x^{\prime}-\frac{t_{0}^{2}}{2} \mu H\left(x_{0}\right) K_{0} \varepsilon|\ln (\varepsilon)| \\
& -\int_{\Omega} F\left(x, t u_{\varepsilon, x_{0}}\right) d x+o(\varepsilon|\ln (\varepsilon)|) \\
& \leq \max _{t \geq 0} J\left(t U_{\varepsilon, 0}\right)-\frac{t_{0}^{2}}{2} \mu H\left(x_{0}\right) p\left(x_{0}\right) K_{0} \varepsilon|\ln (\varepsilon)|-\int_{\Omega} F\left(x, t u_{\varepsilon, x_{0}}\right) d x+o(\varepsilon|\ln (\varepsilon)|) .
\end{aligned}
$$

Therefore

$$
\begin{aligned}
\Phi\left(t_{\varepsilon} u_{\varepsilon, x_{0}}\right) & \leq M_{1}\left(S, S_{1}\right)-\int_{\Omega} F\left(x, t u_{\varepsilon, x_{0}}\right) d x+o(\mu) \\
& -\mu H\left(x_{0}\right) \begin{cases}{\left[\frac{t_{0}^{2}}{2} p\left(x_{0}\right) K_{1}-\frac{t_{0}^{2 *}}{2^{*}} K_{2}+\frac{t_{0}^{2 *}}{2_{*}^{*}} p\left(x_{0}\right) Q\left(x_{0}\right) K_{3}\right] \varepsilon+o(\varepsilon)} & \text { if } N \geq 4 \\
\frac{t_{0}^{2}}{2} p\left(x_{0}\right) K_{0} \varepsilon|\ln (\varepsilon)|+o(\varepsilon|\ln (\varepsilon)|) & \text { if } N=3 .\end{cases}
\end{aligned}
$$

Now, we need to give a explicit form of $t_{0}$. Since $\sup _{t \geq 0} \Phi\left(t u_{\varepsilon, x_{0}}\right)=\sup _{t \geq 0} h(t)$ is achieved at $t_{\varepsilon}$ then $h^{\prime}\left(t_{\varepsilon}\right)=0$ and letting $\varepsilon \rightarrow 0$ we get

$$
\int_{R_{+}^{N}}\left|\nabla U_{1,0}\right|^{2} d x-\frac{t_{0}^{2^{*}-2}}{p\left(x_{0}\right)} \int_{\mathbb{R}_{+}^{N}}\left|U_{1,0}\right|^{2^{*}} d x-Q\left(x_{0}\right) t_{0}^{2_{*}-2} \int_{R^{N-1}}\left|U_{1,0}\right|^{2 *} d x^{\prime}=0 .
$$

On the other hand, since $U_{1,0}$ is a solution of (3.31) we see that

$$
\int_{R_{+}^{N}}\left|\nabla U_{1,0}\right|^{2} d x-N(N-2) \int_{\mathbb{R}_{+}^{N}}\left|U_{1,0}\right|^{2^{*}} d x-\mu \int_{R^{N-1}}\left|U_{1,0}\right|^{2 *} d x^{\prime}=0 .
$$

Combining (3.42) and (3.43) we obtain $t_{0}=\left(p\left(x_{0}\right) N(N-2)\right)^{\frac{1}{2^{*}-2}}$.

Using (3.38) and (3.39), for $N \geq 4$, we see that

$$
\begin{aligned}
\frac{t_{0}^{2}}{2} p\left(x_{0}\right) K_{1}-\frac{t_{0}^{*}}{2^{*}} K_{2}+\frac{t_{0}^{2 *}}{2_{*}} p\left(x_{0}\right) Q\left(x_{0}\right) K_{3} & =p\left(x_{0}\right) \frac{(N-2)^{2}}{2}\left(\frac{4}{N-3}+\frac{2(N-1)}{(N-3)(N-2)^{2}} \mu^{2}\right) \\
& +2 \frac{(N-1)}{(N-2)} \sqrt{N(N-2)} \sqrt{p\left(x_{0}\right)} p\left(x_{0}\right) Q\left(x_{0}\right) \mu \\
& =K>0 .
\end{aligned}
$$

Combining this with (3.41) we obtain

$$
\Phi\left(t_{\varepsilon} u_{\varepsilon, x_{0}}\right) \leq M_{1}\left(S, S_{1}\right)-\int_{\Omega} F\left(x, t u_{\varepsilon, x_{0}}\right) d x-\mu H\left(x_{0}\right) \begin{cases}K \varepsilon+o(\varepsilon) & \text { if } N \geq 4 \\ K_{0} \varepsilon|\ln (\varepsilon)|+o(\varepsilon|\ln (\varepsilon)|) & \text { if } N=3 .\end{cases}
$$

Using (3.1) and the fact that $\int_{\Omega}\left|u_{\varepsilon, x_{0}}\right|^{2} d x=o(\varepsilon),(3.44)$ becomes

$$
\Phi\left(t_{\varepsilon} u_{\varepsilon, x_{0}}\right) \leq M_{1}\left(S, S_{1}\right)-\int_{\Omega} G\left(x, t u_{\varepsilon, x_{0}}\right) d x-\mu H\left(x_{0}\right) \begin{cases}K \varepsilon+o(\varepsilon) & \text { if } N \geq 4 \\ K_{0} \varepsilon|\ln (\varepsilon)|+o(\varepsilon|\ln (\varepsilon)|) & \text { if } N=3 .\end{cases}
$$

where $G(x, s)=\int_{0}^{s} g(x, r) d r$.

Now, we are able to give sufficient conditions on $f$ to have the condition (3.7): 


\section{Proposition 3.3}

Assume that $f(x, u)$ satisfies (3.1)-(3.5) and that $H\left(x_{0}\right)>0$. Suppose that there exists some continuous function $g($.$) such that g(x, u) \geq g(u)$ for $a$. e. $x \in \Omega$ and for all $u \in \mathbb{R}$ and the primitive $G(u)=\int_{0}^{u} g(t) d t$ satisfies :

$\lim _{\varepsilon \rightarrow 0} \varepsilon^{N-1} \int_{\frac{\mu}{N-2}}^{+\infty}\left(1+t^{2}\right)^{\frac{N-1}{2}} \int_{0}^{+\infty} G\left(\frac{\varepsilon^{-\frac{N-2}{2}}}{\left(1+t^{2}\right)^{\frac{N-2}{2}}\left(1+r^{2}\right)^{\frac{N-2}{2}}}\right) r^{N-2} d r d t=0 \quad$ for $N \geq 4$,

and

$$
\lim _{\varepsilon \rightarrow 0} \frac{\varepsilon^{2}}{|\ln (\varepsilon)|} \int_{\frac{\mu}{N-2}}^{+\infty}\left(1+t^{2}\right) \int_{0}^{+\infty} G\left(\frac{\varepsilon^{-\frac{1}{2}}}{\left(1+t^{2}\right)^{\frac{1}{2}}\left(1+r^{2}\right)^{\frac{1}{2}}}\right) r d r d t=0 \quad \text { for } N=3 .
$$

Then condition (3.7) holds.

\section{Proof.}

The proof become directly from (3.45).

\section{Example of $f$ :}

All the assumptions of Proposition 3.3 are satisfied for the following functions:

1. $g(x, u)=g(u)= \pm|u|^{r-2} u \quad$ with $2<r<2 *$ and $u \in \mathbb{R}$.

2. $g(x, u)=g(u)=\frac{u^{2 *-1}\left(2_{*} \ln (u)-1\right)}{(\ln (u))^{2}} \quad$ for $u>0$.

\section{References}

[1] Adimurthi and G. Mancini, The Neumann problem for elliptic equation with critical non linearity, 65th birthday of Prof. Prodi, Scoula Normale superiore, Pisa, Ed. by Ambrosetti and Marino (1991).

[2] A. Ambrosetti and P. Rabinowitz, Dual variational methods in critical point theory and applications, J. Funct. Anal. 14, 1973, pp. 349-381.

[3] Adimurthi and S. L. Yadava, Positive solution for Neumann problem with critical non linearity on boundary, Comm. In Partial Diff. Equations, 16, (11), 1991, pp 1733-1760.

[4] H. Brezis and L. Nirenberg, Positive solutions of nonlinear elliptic equations involving critical Sobolev exponents. Comm. Pure Appl. Math. 36 (1983), no. 4, pp 437-477.

[5] H. Brezis and E. Lieb, A relation between pointwise convergence of functions and convergence of functionals. Proc. Amer. Math. Soc. 88 (1983), no. 3, pp 486-490.

[6] P. Cherrier, Problèmes de Neumann non linéaires sur les variétés Riemanniennes, J. Funct. Anal., 57, (1984), 154-206. 
[7] R. Crouau, R. Hadiji and R. Lewandowski, Critical Sobolev exponent and the dimension three, Houston J. of Math. 18, no 2, (1992), pp 189-204.

[8] J. Chabrowski and B. Ruf, On the critical Neumann problem with perturbations of lower order, Acta Math. Appl. Sin-Engl. Ser., vol 24, N 3, (2008), pp 441-451.

[9] J. Chabrowski and M. Willem, Least energy solutions of a critical Neumann problem with weight, Calc. Var. 15 (2002), pp 421-431.

[10] J. Chabrowski and J. Yang, Sharp Sobolev Inequality Involving a Critical Nonlinearity on a Boundary, Top. Meth. in Nonlinear Analysis, vol 25, N 1, (2005), pp $135-155$.

[11] C. Chabrowski, S. Filippas and A. Tertikas, Positive solutions of a Neumann problem with competing critical nonlinearities, Topolog. Meth. Nonlin. Analysis 28 (2006), pp 1-32.

[12] M. Chipot, M. Fila and I. Shafrir, On the solutions to some elliptic equations with nonlinear Neumann boundary conditions. Adv. Diff. Eqns 1, (1996), pp 91-110.

[13] J. F. Escobar, Sharp constant in a Sobolev trace inequality, Indiana Univ. Math. J., 37 (1988), pp 687-698.

[14] J. F. Escobar, Uniqueness theorems on conformal deformation of Riemannian metric to a scalar flat metric with constant mean curvature, Annals of Mathematics, 136 (1992), pp 1-50.

[15] R. Hadiji, Solutions positives de l'équation $-\Delta u=u^{p}+\mu u^{q}$ dans un domaine à trou, Ann. Fac. Sci. Toulouse Math. (5) 11, no 3, 1(990), pp 55-71.

[16] R. Hadiji and H. Yazidi, Problem with critical Sobolev exponent and with weight, Chinese Annals of Mathematics, Serie B, 28, no 3, (2007), pp 327-352.

[17] R. Hadiji, R. Molle, D. Passaseo and H. Yazidi, Localization of solutions for nonlinear elliptic problems with critical growth, Comptes Rendus de l'Académie de Sciences (Paris), Ser.I.343, (2006), pp 725-730.

[18] P. L. Lions, The concentration-compactness principle in the calculus of variations. The limit case, Part 1, Revista Mat. Iberoamericana 1, no 1, (1985), pp. 145-201 ; Part 2, Revista Mat. Iberoamericana 1, no 2, (1985), pp. 45-121.

[19] D. Pierotti and S. Terracini, On a Neumann problem involving two critical exponents: remarks on geometrical and topological aspects, Calc. Var. PDEs, 5 (1997), pp 271-291.

[20] M. Struwe, A global compactness result for elliptic boundary value problems involving limiting nonlinearities, Math. Z., 187, (1984), pp. 511-517. 
[21] H. Yazidi, On some nonlinear Neumann problem with weight and with critical Sobolev trace maps, Proceedings of the Royal Society of Edinburgh, 137 A, (2007), pp 1-24.

[22] H. Yazidi, On nonhomogeneneous Neumann problem with weight and with critical nonlinearity in the boundary, Nonlinear Analysis: Theory, Methods and Applications, series A, vol. 68, Issue 2, (2008), pp 329-364.

[23] Y. Y. Li and M. Zhu, Uniqueness theorems through the method of moving spheres. Duke Math. J., 80, (1995), pp 383-417.

[24] M. Zhu, Sharp Sobolev inequality with interior norm, Calc. Var. 8, (1999), pp 27-43. 